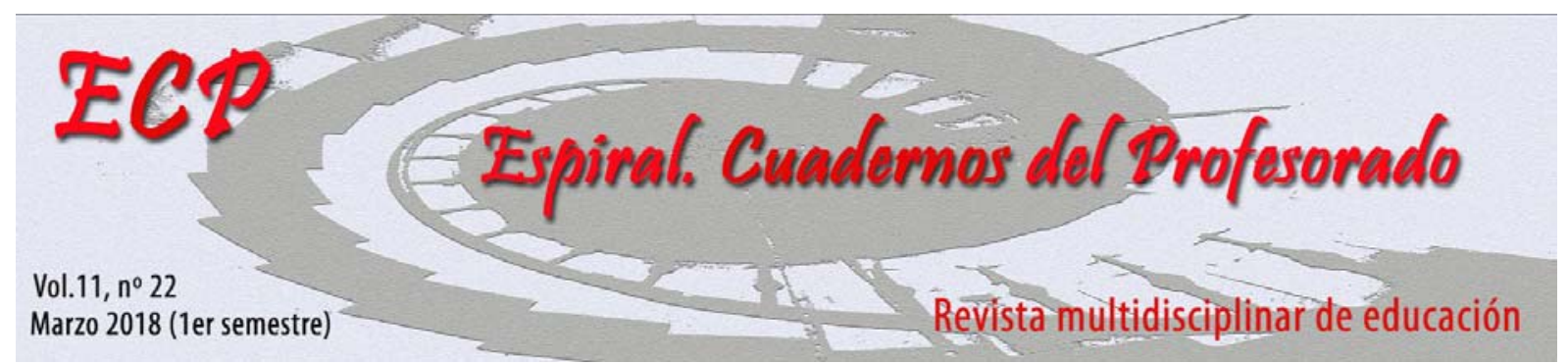

\title{
Mejora de la motivación y las metas sociales tras la realización de una unidad didáctica de escalada
}

\section{Improvement of motivation and social goals after the realization of a didactic unit of climbing}

\author{
Pascual Juliá Hurtado y Antonio Baena-Extremera \\ Facultad de Ciencias de la Educación, Universidad de Granada, España
}

\begin{abstract}
RESUMEN: El objetivo del trabajo fue observar si se producían cambios significativos en la satisfacción, metas sociales y motivación en las clases de Educación Física, tras utilizar una Unidad Didáctica de escalada. Este trabajo se trata de una investigación con un diseño cuasi experimental con medidas pre post y con carácter descriptivo. Se utilizó una muestra de 66 participantes (34 chicas y 32 chicos) de un centro de Educación Primaria de la provincia de Granada, con edades entre 10 y 13 años $(M=11 ; D T=.84)$. Como instrumentos se han utilizados las escalas Sport Motivation Scale (SMS), la Escala de Metas Sociales en Educación Física (EMSEF) y la Escala de Satisfacción en Educación Física (SSI). Se realizó un análisis de consistencia interna, la prueba $t$ para muestras relacionadas y ANOVA. Los resultados nos han mostrado que una vez realizada la Unidad Didáctica, los factores han tenido un cambio significativo y positivo.
\end{abstract}

Palabras clave: Motivación, metas sociales, educación física.

ABSTRACT: The aim of this project was observed if there were some significant changes in some aspects like: satisfaction, social goals and motivation in lessons of Physical Education, after using a teaching unit of rise. This work is a research with a quasi-experimental design with pre post and descriptive measures. A sample of 66 participants was using (34 girls and 32 boys) of a Primary School of Education in Granada, between 10 and 13 years old $(M=11 ; S D=.84)$. We have used some instruments like: Sport Motivation Scale (SMS), Scale of Socials Goals in Physical Education (EMSEF) and the Scale of Satisfaction in Physical Education (SSI). Was performed an analysis of internal consistency, a proof $t$ for samples associated and ANOVA was performed. The results have shown that when the teaching unit has finished, the factors have had a significant change and positive.

Key words: Motivation, social goals, physical education.

Juliá Hurtado, P. y Baena-Extremera, A. (2018). Mejora de la motivación y las metas sociales tras la realización de una unidad didáctica de escalada. Espiral. Cuadernos del Profesorado, 11(22), 46-51.

Fecha de recepción: 23/04/2017

Fecha de aceptación: 03/10/2017
Correspondencia: pascualjulia94@gmail.com

(P. Juliá Hurtado) 


\section{Introducción}

En los últimos años cada vez se han ido realizando más investigaciones relacionadas con la Educación Física (EF) y la motivación que despierta en los alumnos esta asignatura, puesto que, según Inglés, Martínez-González, Torregrosa, García-Fernández, y Ruiz-Esteban (2011), la motivación es un factor muy importante y de gran interés debido a su importancia en el éxito académico de los alumnos.

Existen diferentes teorías motivacionales que son relevantes para esta investigación, de las cuales destacamos la Teoría de la Autodeterminación (Ryan y Deci, 2000) y la teoría de las metas de logro de Duda y Nicholls (1989), puesto que son dos de las teorías más utilizadas por los investigadores actualmente para casos de este tipo (estudios motivacionales).

En primer lugar, la Teoría de la Autodeterminación, se trata de un modelo que explica la motivación de los seres humanos en distintos ámbitos. La teoría analiza el grado en que las personas desarrollan sus acciones al nivel más alto y se involucran en las acciones de manera voluntaria (Ryan y Deci, 2000).

En segundo lugar, para lograr una mejor comprensión de la motivación debemos entender la Teoría de las metas de logro. La idea principal de la teoría es que todas las personas somos seres con ciertas intenciones, guiadas por nuestros objetivos de modo que actuamos de acuerdo con ellos (Nicholls, Cheung, Lauer, y Patashnick, 1989).

Sin embargo la motivación no se puede limitar a lo mencionado antes en la Teoría de las metas de logro ya que diferentes investigaciones (Anderman y Anderman, 1999) han demostrado que los alumnos pueden tener ciertas razones sociales para establecer su éxito.

Para tratar estos dos temas, tanto en la motivación como en las metas sociales de los alumnos, existen ciertos instrumentos de medida, los cuales son: para medir la motivación en educación, utilizamos la Sport Motivation Scale (SMS), escala que en un principio fue desarrollada para la motivación en el deporte (Échelle de Motivation dans les Sports, de Brière, Vallerand, Blais, y Pelletier, 1995), y posteriormente se adaptó al contexto educativo dando lugar finalmente a la SMS, instrumento muy utilizado en las últimas investigaciones (Granero-Gallegos, Baena-Extremera, Gómez-López, y Abraldes, 2013).

En cuanto a las metas sociales en relación con la EF, utilizaremos la Escala de Metas Sociales en Educación Física (EMSEF), se trata de una adaptación a partir de la Social Goal Scale-Physical Education (SGS-PE) de Guan, Xiang, y McBride (2005), adaptada a la EF por Moreno-Murcia, Coll, y Camacho (2007).

El objetivo de esta investigación es observar en nivel de satisfacción, las metas sociales y la motivación de los alumnos, y descubrir si esto puede cambiar con Unidades Didácticas diferentes y actividades novedosas, en este caso la escalada. Existen ya evidencias de cambios en diversas variables psicológicas tras aplicar ciertos contenidos de actividades de aventura y en la naturaleza (Baena-Extremera y Granero-Gallegos, 2013; Baena-Extremera, Granero-Gallegos, y Ortiz-Camacho, 2011).

Teniendo en cuenta todo lo anterior, y el conocimiento ya existente sobre la posibilidad de modificar ciertas variables psicoeducativas en el alumnado tras impartir ciertos contenidos innovadores, en este caso una Unidad Didáctica de escalada, el objetivo de este trabajo es observar si se producen cambios positivos tanto en la motivación como en las metas sociales de los alumnos una vez realizadas las sesiones de escalada.

\section{Material y método}

\section{Participantes}

En esta investigación han participado un total de 66 alumnos de quinto y sexto de primaria, de los cuales 32 han sido chicos $(48,5 \%)$ y 34 chicas $(51,5 \%)$, todos del mismo centro de Primaria en la provincia de Granada. El rango de edad de los participantes está entre 10 y $13(M=11 ; D T=.84)$. 


\section{Diseño}

Este trabajo se trata de una investigación con un diseño cuasi experimental con medidas pre post y con carácter descriptivo. El diseño cuasi experimental se trata de un modo de investigación donde los grupos que se utilizan para probar una variable sobre ellos, no están seleccionados de forma aleatoria, puesto que se tratan de grupos que ya están hechos. Ha sido utilizado con unas medidas descriptivas y pre post, tratando de obtener una visión global del objetivo mencionados en la introducción, observando si se han producido cambios en el antes y después de la investigación. Este modelo de investigación descriptiva suelen dar resultados abiertos, siendo una herramienta útil para posteriores investigaciones.

\section{Instrumentos}

Para la realización de esta investigación se han utilizado diversas escalas para medir la motivación, metas sociales y satisfacción de los alumnos durante las clases de Educación Física.

Se ha utilizado la Sport Satisfaction Instrument (SSI) (Duda y Nicholls, 1992) en su versión española adaptada a la Educación Física (Baena-Extremera, Granero-Gallegos, Bracho-Amador y Perez-Quero, (2012), puesto que el objetivo de estudio de la SSI es el deporte, mientras que el de esta investigación es en la E.F. La SSI-EF adaptada consta de ocho ítems para medir la satisfacción en las clases de educación física, dividiendo estos ocho ítems en dos partes, una que se encarga de medir la satisfacción y diversión (5 ítems) y por otro lado el aburrimiento (3 ítems). En el cuestionario, los alumnos deben reflejar el nivel o grado de acuerdo, que tienen respecto a los ítems que se plantean. Las respuestas, están planteadas mediante una escala de tipo Likert, siendo de cinco puntos que varían del 1 al 5 siendo 1 "muy en desacuerdo" y 5 "muy de acuerdo". Ha este instrumento se le ha realizado un análisis de fiabilidad (alfa de Cronbach) en cada factor (satisfacción/diversión y aburrimiento) para comprobar la fiabilidad de los cuestionarios realizados por los participantes, dando como resultado un valor alfa de 29 .

Por otro lado, para medir las metas sociales se ha utilizado la Escala de Metas Sociales en Educación Física (EMSEF), en su versión en español de la Social Goal Scale-Physical Education (SGS-PE) (Guan et al. 2005), de los autores Moreno-Murcia et al., 2007. Este instrumento consta de once ítems, divididos en dos factores donde se mide por un lado las metas de relación (6 ítems) y por otro lado las metas de responsabilidad (5 ítems). Las respuestas, al igual que la anterior, están diseñadas con la escala de tipo Likert, pero en este caso de siete puntos, donde varían del 1 al 7, siendo 1 "totalmente en desacuerdo" y 7 "totalmente de acuerdo". En esta herramienta el valor alfa obtenido ha sido en el factor responsabilidad de $.67 \mathrm{y}$ en el factor relación de .76.

Finalmente la última herramienta que ha sido utilizada para esta investigación ha sido la Sport Motivation Scale (SMS) (Brière et al., 1995) en su versión española realizada por Balaguer, Castillo y Duda (2007) y adaptada a la EF (Granero-Gallegos et al., 2013). Esta escala está compuesta por veintiocho ítems, subdivididos en diferentes partes, donde se miden distintos aspectos de la motivación: la motivación intrínseca, la motivación extrínseca y la amotivación. Cada ítem está encabezado por una primera frase "Participo y me esfuerzo en las clases de Educación Física". Las respuestas del cuestionario también están puntuadas en una escala de tipo Likert entre 1 "totalmente en desacuerdo" y 7 "totalmente de acuerdo". El valor alfa obtenido en cada factor de este instrumento ha sido bastante altos, el alfa obtenido en el factor de motivación intrínseca ha sido de .82, en el factor de motivación extrínseca ha sido de $.80 \mathrm{y}$ en el de amotivación de .65 .

\section{Proceso de adaptación}

En esta investigación ha sido necesario realizar unas pequeñas modificaciones en las cuestiones que se planteaban para lograr una mejor comprensión de los estudiantes, sin alterar la esencia de las preguntas. Las cuestiones originales que han sido modificadas son las siguientes: de la SSI la cuestión número 6 (Cuando hago Educación Física parece que el tiempo vuela). De la SMS se han modificado la pregunta 2 (Participo y me esfuerzo en las clases de Educación Física por el placer de saber más sobre las actividades que practico), la pregunta 4 (Participo y me esfuerzo en las clases de Educación Física por el placer de descubrir nuevas actividades físico-deportivas), la pregunta 5 
(Participo y me esfuerzo en las clases de Educación Física tengo la impresión de que no soy capaz de tener éxito en las actividades físico-deportivas que realizo), la pregunta 8 (Participo y me esfuerzo en las clases de Educación Física porque me siento muy satisfecho cuando consigo realizar adecuadamente las actividades físico-deportivas más difíciles), la pregunta 10 (Participo y me esfuerzo en las clases de Educación Física por el prestigio de ser bueno en las actividades de clase) y la pregunta 27 (Participo y me esfuerzo en las clases de Educación Física por el placer de descubrir nuevas estrategias de ejecución).

A continuación se muestran las cuestiones que han sido modificadas con sus respectivos cambios: de la SSI la pregunta número 6 (Cuando hago Educación Física parece que el tiempo pasa muy deprisa). De la SMS la pregunta 2 (Participo y me esfuerzo en las clases de Educación Física para saber más sobre las actividades que realizamos en clase), la pregunta 4 (Participo y me esfuerzo en las clases de Educación Física para descubrir nuevas actividades y deportes), la pregunta 5 (Pienso que no puedo tener éxito en las actividades deportivas que realizo), la pregunta 8 (Participo y me esfuerzo en las clases de Educación Física porque me siento muy satisfecho cuando consigo realizar adecuadamente las actividades deportivas que realizamos en clase), la pregunta 10 (Participo y me esfuerzo en las clases de Educación Física para ser el mejor en las actividades de clase) y la pregunta 27 (Participo y me esfuerzo en las clases de Educación Física por el placer de aprender nuevas formas de hacer las actividades deportivas).

Por otro lado las escalas de Likert utilizadas en todos los instrumentos también han sido modificadas, puesto que en vez de puntuarlas numéricamente, han sido puntuadas mediante caras de emoticonos con diferentes expresiones, como ya se hizo en otros trabajos de investigación de este tipo, (Berrocal y Pacheco, 2005; Arias, Castejón, y Yuste, 2013), siendo la cara más triste "totalmente en desacuerdo" y la cara más alegre "totalmente de acuerdo", de este modo les resulta más sencillo interpretar y responder las cuestiones a los alumnos.

\section{Procedimiento}

Para realizar la investigación se ha obtenido el permiso por parte del órgano de dirección del centro educativo. Los estudiantes fueron informados de la finalidad del estudio. Se solicitó a los estudiantes una autorización por escrito de parte de sus padres para que pudieran participar en la investigación, puesto que son menores de edad. Previamente a la investigación, las escalas que se han utilizado fueron administradas a un grupo reducido de alumnos (15 estudiantes) para comprobar su correcta comprensión, y en caso contrario llevar a cabo ciertos cambios pertinentes, nombrados anteriormente en el proceso de adaptación. Posteriormente, se pasó la escala a los educandos durante la hora correspondiente de Educación Física.

\section{Análisis de datos}

Se llevó a cabo un análisis de consistencia interna (Alfa de Cronbach) con el objetivo de obtener la fiabilidad de las escalas y los factores creados (Aburrimiento, relación, motivación intrínseca, motivación extrínseca y amotivación). Posteriormente, para obtener las diferencias entre el pre y post, se efectuó la prueba t de Student para muestras relacionadas y Anova por sexo.

\section{Resultados}

\section{Análisis de fiabilidad de la escala}

En la Tabla 1 se puede observar los análisis estadísticos descriptivos pre y post por ítem de la SSI adaptada a la EF. Se ha mantenido el ítem-factor que viene definido en el instrumento primario (Duda y Nicholls, 1992) y en la versión de Baena-Extremera et al. (2012).

En la Tabla 2 se muestran los datos resultantes del análisis estadístico descriptivo pre y post de los ítems de la EMSEF, manteniendo el ítem-factor del SGE-PE (Guan, Xiang y McBride, (2005), y de la versión española de Moreno-Murcia et al., 2007 siendo estos factores totalmente adecuados para la EF. En la Tabla 3 se observan los análisis estadísticos descriptivos pre post por ítem de la SMS, se ha mantenido el ítem-factor establecido en su adaptación a la EF (Granero-Gallegos, BaenaExtremera, Gómez-López y Abraldes, 2013). 
Tabla 1.

Análisis estadísticos descriptivos pre post de la SSI por ítem.

\begin{tabular}{|c|c|c|c|c|}
\hline & \multicolumn{2}{|c|}{ PRE } & \multicolumn{2}{|c|}{ POST } \\
\hline & $M$ & $S D$ & $M$ & $S D$ \\
\hline \multicolumn{5}{|l|}{ Satisfacción/diversión } \\
\hline 1. Normalmente me divierto en las clases de Educación Física & 4.74 & .53 & 4.89 & .31 \\
\hline 5. Normalmente encuentro la Educación Física interesante & 4.24 & 1.15 & 4.12 & 1.20 \\
\hline $\begin{array}{l}\text { 6. Cuando hago Educación Física parece que el tiempo pasa muy } \\
\text { deprisa }\end{array}$ & 3.89 & 1.5 & 4.23 & 1.13 \\
\hline $\begin{array}{l}\text { 7. Normalmente participo activamente en las clases de Educación } \\
\text { Física }\end{array}$ & 4.36 & 1.09 & $4 . .2$ & .78 \\
\hline 8. Normalmente me lo paso bien haciendo Educación Física & 4.91 & .29 & 4.91 & .29 \\
\hline \multicolumn{5}{|l|}{ Aburrimiento } \\
\hline $\begin{array}{l}\text { 2. En las clases de Educación Física a menudo sueño despierto en } \\
\text { vez de pensar en lo que hago realmente }\end{array}$ & 2.53 & 1.35 & 2 & .99 \\
\hline 3. En las clases de Educación Física, normalmente me aburro & 2 & 1.46 & 1.48 & .74 \\
\hline 4. En Educación Física deseo que la clase termine rápidamente & 1.70 & 1.32 & 1.41 & .67 \\
\hline
\end{tabular}

Nota: Media (M) y desviación típica (SD).

Tabla 2.

Análisis estadísticos descriptivos pre post de la EMSEF por ítem.

\begin{tabular}{|c|c|c|c|c|}
\hline & \multicolumn{2}{|c|}{ PRE } & \multicolumn{2}{|c|}{ POST } \\
\hline & $M$ & $S D$ & $M$ & $S D$ \\
\hline \multicolumn{5}{|l|}{ Responsabilidad } \\
\hline 1. Intento hacer lo que el maestro me pide que haga & 6.12 & 1.35 & 6 & 1.32 \\
\hline 3. Es importante para mí seguir las reglas de clase & 6.55 & .98 & 6.09 & 1.26 \\
\hline $\begin{array}{l}\text { 5. Es importante para mí seguir trabajando incluso cuando otros } \\
\text { compañeros no están haciendo nada }\end{array}$ & 5.83 & 1.73 & 5.70 & 1.65 \\
\hline $\begin{array}{l}\text { 7. Me gustaría que el profesor pensara que soy un/a estudiante } \\
\text { responsable }\end{array}$ & 6.36 & 1.07 & 6 & 1.24 \\
\hline $\begin{array}{l}\text { 9. No me gustaría distraer a un/a compañero/a de clase cuando } \\
\text { está realizando una actividad individual }\end{array}$ & 4.97 & 2.40 & 4.71 & 2.18 \\
\hline \multicolumn{5}{|l|}{ Relación } \\
\hline 2. Me gustaría llegar a conocer muy bien a mis amigos de clase & 5.94 & 1.73 & 6.36 & 1.26 \\
\hline $\begin{array}{l}\text { 4. Me gustaría mantener las promesas que he hecho a otros/as } \\
\text { compañeros/as en mi clase. }\end{array}$ & 5.67 & 1.85 & 6.53 & .94 \\
\hline 6. Me gustaría tener un/a amigo/a en el que poder confiar & 6.41 & 1.52 & 6.53 & 1.05 \\
\hline $\begin{array}{l}\text { 8. Me gustaría llevarme bien con la mayoría de los otros } \\
\text { estudiantes }\end{array}$ & 6.48 & 1.15 & 6.56 & .97 \\
\hline 10. Es importante para mí ser aceptado/a por otros/as estudiantes & 6.11 & 1.52 & 6.36 & 1.23 \\
\hline $\begin{array}{l}\text { 11. Es importante para mí tener uno/a o dos amigos/as muy } \\
\text { íntimos }\end{array}$ & 6.05 & 1.46 & 6.36 & 1.06 \\
\hline
\end{tabular}

Nota: Media (M) y desviación típica (SD). 
Tabla 3.

Análisis estadísticos descriptivos pre post de la SMS por ítem.

\begin{tabular}{|c|c|c|c|c|}
\hline & \multicolumn{2}{|c|}{ PRE } & \multicolumn{2}{|c|}{ POST } \\
\hline & $M$ & $S D$ & $M$ & $S D$ \\
\hline \multicolumn{5}{|l|}{ M.I. } \\
\hline 1. Por el placer de vivir experiencias estimulantes & 6.20 & 1.21 & 6.86 & .42 \\
\hline 2. Para saber más sobre las actividades que realizamos en clase & 6 & 1.51 & 6.74 & .66 \\
\hline 4. Para descubrir nuevas actividades y deportes & 6.27 & 1.11 & 6.56 & .96 \\
\hline $\begin{array}{l}\text { 8. Porque me siento muy satisfecho cuando consigo realizar } \\
\text { adecuadamente las actividades deportivas que realizamos en clase }\end{array}$ & 5.86 & 1.66 & 6.64 & .87 \\
\hline 12. Por el placer que siento cuando mejoro alguno de mis puntos débiles. & 6.05 & 1.40 & 6.44 & 1.24 \\
\hline $\begin{array}{l}\text { 13. Por la sensación que tengo cuando estoy concentrado realmente en la } \\
\text { actividad }\end{array}$ & 5.70 & 1.62 & 6.47 & 1.04 \\
\hline 15. Por la satisfacción que tengo cuando estoy mejorando mis habilidades & 5.71 & 1.28 & 6.52 & .96 \\
\hline $\begin{array}{l}\text { 18. Por las intensas emociones que experimento cuando practico una } \\
\text { actividad físico-deportiva que me gusta }\end{array}$ & 6.17 & 1.28 & 6.65 & .71 \\
\hline 20. Por el placer que siento mientras realizo ciertos movimientos difíciles & 5.70 & 1.38 & 6.56 & .84 \\
\hline $\begin{array}{l}\text { 23. Por el placer que siento cuando aprendo a realizar actividades que } \\
\text { nunca había hecho anteriormente }\end{array}$ & 5.92 & 1.48 & 6.68 & .78 \\
\hline $\begin{array}{l}\text { 25. Porque me gusta el sentimiento de estar totalmente metido en la } \\
\text { actividad }\end{array}$ & 5.82 & 1.64 & 6.58 & .99 \\
\hline $\begin{array}{l}\text { 27. Por el placer de aprender nuevas formas de hacer las actividades } \\
\text { deportivas }\end{array}$ & 5.98 & 1.57 & 6.76 & .58 \\
\hline \multicolumn{5}{|l|}{ M.E. } \\
\hline 6. Porque me permite estar bien considerado entre la gente que conozco & 5.77 & 1.67 & 5.56 & 1.59 \\
\hline $\begin{array}{l}\text { 7. Porque, en mi opinión, es una de las mejores formas de relacionarme con } \\
\text { los demás }\end{array}$ & 5.41 & 1.85 & 5.29 & 1.75 \\
\hline 9. Porque es una manera de estar en forma & 5.97 & 1.64 & 5.82 & 1.34 \\
\hline 10. Para ser el mejor en las actividades de clase & 5.33 & 1.98 & 5.14 & 1.94 \\
\hline $\begin{array}{l}\text { 11. Porque es una de las mejores formas de desarrollar otros aspectos de mí } \\
\text { mismo }\end{array}$ & 5.65 & 1.45 & 5.52 & 1.35 \\
\hline $\begin{array}{l}\text { 14. Porque debo practicar actividad físico-deportiva para sentirme bien } \\
\text { conmigo mismo }\end{array}$ & 5.82 & 1.60 & 5.32 & 1.53 \\
\hline $\begin{array}{l}\text { 16. Porque las personas de mí alrededor piensan que es importante estar en } \\
\text { forma }\end{array}$ & 5.06 & 2.14 & 4.85 & 1.87 \\
\hline $\begin{array}{l}\text { 17. Porque es una buena forma de aprender cosas que me pueden ser útiles } \\
\text { en otros aspectos de mi vida }\end{array}$ & 6.06 & 1.38 & 5.64 & 1.44 \\
\hline 21. Porque me sentiría mal si no participase en la clase & 5.27 & 2.06 & 5.06 & 1.74 \\
\hline 22. Para mostrar a los demás lo bueno que soy cuando hago las actividades & 5.20 & 2.15 & 5.12 & 1.86 \\
\hline $\begin{array}{l}\text { 24. Porque es una de las mejores formas de mantener buenas relaciones con } \\
\text { mis amigos/as }\end{array}$ & 5.67 & 1.72 & 5.05 & 1.63 \\
\hline 26. Porque debo adquirir hábitos de práctica físico-deportiva & 5.53 & 1.57 & 5.26 & 1.35 \\
\hline \multicolumn{5}{|l|}{ Amotivación } \\
\hline $\begin{array}{l}\text { 3. Antes participaba y me esforzaba en las clases, pero ahora me pregunto } \\
\text { si debo continuar haciéndolo. }\end{array}$ & 4.80 & 2.38 & 3.73 & 2.29 \\
\hline 5. Pienso que no puedo tener éxito en las actividades deportivas que & 3.83 & 2.40 & 2.97 & 2.18 \\
\hline
\end{tabular}
realizo. 
19. Realmente no me siento capacitado para la práctica físico-deportiva

28. A menudo me digo a mi mismo/a que no puedo alcanzar las metas que me establezco

$\begin{array}{llll}3.48 & 2.43 & 2.80 & 1.86 \\ 3.71 & 2.23 & 1.86 & 1.06\end{array}$

Nota: Media (M), desviación típica (SD) motivación intrínseca (M.I.) y motivación extrínseca (M.E.).

El primer factor de la SSI (satisfacción/diversión) presentó un valor de fiabilidad bastante bajo en el pre como en el post, dando un alfa de .17, por ello este factor no ha sido tenido en cuenta para la comparación de medias. El segundo factor de la SSI (Aburrimiento) presentó un valor de fiabilidad de .56 , teniendo en cuenta que este factor solamente consta de tres ítems, este valor alfa es asumible y se tiene en cuenta para los análisis. El primer factor de la EMSEF (Responsabilidad) presentó un valor alfa de .67 en el post, mientras que en el pre dio un alfa de Cronbach bajo, lo cual da como resultados datos no significativos. El segundo factor de la EMSEF (Relación) presentó un valor alfa de .76 tanto en el pre como en el post. En cuanto a los factores de la SMS, han presentado un alto grado de fiabilidad, siendo el valor alfa del primer factor (Motivación Intrínseca) .82, el segundo factor (Motivación Extrínseca) $.80 \mathrm{y}$ el tercer factor (Amotivación) .65, este último factor presenta un buen valor, teniendo en cuenta que está compuesto por pocos ítems, al igual que el factor de aburrimiento.

Tabla 4

Medias relacionadas.

\begin{tabular}{lcccc}
\hline \multirow{2}{*}{ Factores } & \multicolumn{2}{c}{ PRE } & \multicolumn{2}{c}{ POST } \\
\cline { 2 - 5 } Aburrimiento & $\boldsymbol{M}$ & $\boldsymbol{S D}$ & $\boldsymbol{M}$ & $\boldsymbol{S D}$ \\
Relación & 2.07 & 1.01 & 1.63 & .57 \\
Motivación Intrínseca & 6.10 & 1.04 & 6.45 & .73 \\
Motivación Extrínseca & 5.94 & .84 & 6.62 & .50 \\
Amotivación & 5.56 & .98 & 5.30 & .91 \\
\hline
\end{tabular}

Nota: Media (M) y desviación típica (SD).

Tabla 5 .

Medias relacionadas por sexo.

\begin{tabular}{lcccccccc}
\hline \multirow{2}{*}{ Factores } & \multicolumn{4}{c}{ PRE } & \multicolumn{2}{c}{ SD } & \multicolumn{3}{c}{ MOST } \\
\cline { 2 - 9 } & \multicolumn{2}{c}{$\boldsymbol{M}$} & \multicolumn{1}{c}{ SD } \\
\cline { 2 - 9 } & Masc. & Fem. & Masc. & Fem. & Masc. & Fem. & Masc. \\
\hline Aburrimiento & 1.96 & 2.17 & .93 & 1.08 & 1.57 & 1.68 & .48 \\
Relación & 5.86 & 6.33 & 1.28 & .68 & 6.34 & 6.55 & .89 \\
Motivación Intrínseca & 6.07 & 5.82 & .59 & 1.02 & 6.72 & 6.52 & .26 \\
Motivación Extrínseca & 5.83 & 5.30 & .75 & 1.10 & 5.37 & 5.22 & .96 \\
Amotivación & 4.21 & 3.71 & 1.79 & 1.50 & 2.96 & 2.72 & 1.37 \\
\hline
\end{tabular}

Nota: Media $(M)$ y desviación típica $(S D)$.

También podemos observar cómo se muestran las medias relacionadas por sexo pre y post en la Tabla 5, con el fin de analizar los efectos de interacción del sexo en los diferentes factores.

En la Tabla 6, se muestra el análisis de las diferencias entre el pre y el post. Destacar que solo se encontraron diferencias en la motivación extrínseca durante el pre $\left(\mathrm{F}_{(4,96)} p=.030\right)$, no encontrándose diferencias significativas ni en el resto de factores y ni en los diferentes momentos. 
Tabla 6.

ANOVA pre y post-test.

\begin{tabular}{|c|c|c|c|c|c|c|}
\hline & & $\begin{array}{c}\text { Suma de } \\
\text { cuadrados }\end{array}$ & gl & $\begin{array}{c}\text { Medias } \\
\text { cuadráticas }\end{array}$ & $\mathbf{F}$ & Sig. \\
\hline \multirow{3}{*}{$\begin{array}{l}\text { Aburrimiento } \\
\text { Pre }\end{array}$} & Intergrupos & .711 & 1 & .711 & .691 & .409 \\
\hline & Intragrupos & 65.910 & 64 & 1.030 & & \\
\hline & Total & 66.621 & 65 & & & \\
\hline \multirow[t]{3}{*}{ Post } & Intergrupos & .212 & 1 & .212 & .641 & .426 \\
\hline & Intragrupos & 21.150 & 64 & .330 & & \\
\hline & Total & 21.362 & 65 & & & \\
\hline \multirow{3}{*}{$\begin{array}{l}\text { Relación } \\
\text { Pre }\end{array}$} & Intergrupos & 3.542 & 1 & 3.542 & 3.379 & .071 \\
\hline & Intragrupos & 67.096 & 64 & 1.048 & & \\
\hline & Total & 70.638 & 65 & & & \\
\hline \multirow[t]{3}{*}{ Post } & Intergrupos & .728 & 1 & .728 & 1.341 & .251 \\
\hline & Intragrupos & 34.759 & 64 & .543 & & \\
\hline & Total & 35.487 & 65 & & & \\
\hline \multirow{3}{*}{$\begin{array}{l}\text { Motivación } \\
\text { Intrínseca } \\
\text { Pre }\end{array}$} & Intergrupos & 1.048 & 1 & 1.048 & 1.467 & .230 \\
\hline & Intragrupos & 45.713 & 64 & .714 & & \\
\hline & Total & 46.761 & 65 & & & \\
\hline \multirow[t]{3}{*}{ Post } & Intergrupos & .623 & 1 & .623 & 2.463 & .121 \\
\hline & Intragrupos & 16.185 & 64 & .253 & & \\
\hline & Total & 16.808 & 65 & & & \\
\hline \multirow{3}{*}{$\begin{array}{l}\text { Motivación } \\
\text { Extrínseca } \\
\text { Pre }\end{array}$} & Intergrupos & 4.490 & 1 & 4.490 & 4.955 & .030 \\
\hline & Intragrupos & 58.000 & 64 & .906 & & \\
\hline & Total & 62.490 & 65 & & & \\
\hline \multirow[t]{3}{*}{ Post } & Intergrupos & .369 & 1 & .369 & .434 & .512 \\
\hline & Intragrupos & 54.449 & 64 & .851 & & \\
\hline & Total & 54.818 & 65 & & & \\
\hline \multirow{3}{*}{$\begin{array}{l}\text { Amotivación } \\
\text { Pre }\end{array}$} & Intergrupos & 4.213 & 1 & 4.213 & 1.537 & .220 \\
\hline & Intragrupos & 175.360 & 64 & 2.740 & & \\
\hline & Total & 179.573 & 65 & & & \\
\hline \multirow[t]{3}{*}{ Post } & Intergrupos & .895 & 1 & .895 & .550 & .461 \\
\hline & Intragrupos & 104.185 & 64 & 1.628 & & \\
\hline & Total & 105.080 & 65 & & & \\
\hline
\end{tabular}

Nota: $\mathrm{gl}=$ grados de liberta; $\mathrm{F}=$ prueba $\mathrm{F}$; $\mathrm{Sig}=$ significatividad. La diferencia de medias es significativa al nivel $p<.05$ 


\section{Efectos de intervención}

Para el análisis de los efectos causados en la intervención, se ha llevado a cabo la prueba $t$ de student para muestras relacionadas. Los datos de los factores relacionados con un pre y post pueden observarse en la Tabla 4, a través de las medias de antes y después. Todos los factores han tenido un cambio significativo. En el factor aburrimiento se han obtenido unos datos con ciertos cambios muy significativo $\left(\mathrm{F}_{(4,65)} p<.001\right)$, donde se aprecia claramente a través de la media en la tabla 4 como el aburrimiento ha descendido ligeramente, lo cual equivale a un menor aburrimiento después de las sesiones. El segundo factor (Relación) también ha tenido un cambio muy significativo $\mathrm{F}_{(3,65)} p<.001$, donde al igual que el anterior podemos apreciar por medio de las medias reflejadas, que la relación entre compañeros aumenta levemente una vez realizadas las sesiones. En cuanto a los factores de Motivación Intrínseca (MI), Motivación Extrínseca y Amotivación posteriores, los datos han confirmado la relación significativa y positiva que se ha obtenido entre los factores, reflejando diferencias estadísticamente significativas en cada uno de los factores, $\mathrm{MI}\left(\mathrm{F}_{(10,65)} p<.001\right)$, $\mathrm{ME}\left(\mathrm{F}_{(2,65)}\right.$ $\mathrm{p}=.007)$ y Amotivación $\left(\mathrm{F}_{(7,65)} p<.001\right)$.

Los alumnos han mostrado un aumento en la relación y motivación intrínseca, y una disminución del aburrimiento, motivación extrínseca y amotivación posteriormente a las sesiones realizadas.

\section{Discusión}

El objetivo de este trabajo ha sido analizar los cambios producidos en los diferentes factores (aburrimiento, relación, motivación intrínseca, motivación extrínseca y amotivación) una vez realizadas las sesiones de escalada con alumnos de educación primaria. Teniendo en cuenta, que ya existen trabajo que muestran cambios tras usar alguna unidad didáctica de contenidos de aventura y medio natural (Baena-Extremera et al., 2011, Baena-Extremera y Granero-Gallegos, 2013).

La realización de este trabajo ha sido muy importante por un motivo fundamental, que no se suelen utilizar los deportes de aventura como recurso en la EF, a pesar de tratarse de un contenido muy motivante e interesante para los alumnos (Baena-Extremera et al., 2011). Este contenido no suele usarse en las clases de EF a pesar de que según el Real Decreto 126/ (2014), de 28 de Febrero, por el que se establece el currículo básico de la Educación Primaria las situaciones adaptadas al entorno físico, están relacionadas con el medio urbano o natural y pueden trabajarse muchos aspectos de forma trasversal, como por ejemplo la seguridad en el medio natural o la profundización de valores al incluir el respeto y conservación del entorno natural, a través de actividades como las salidas a pie o en bicicleta, el esquí o la escalada entre otras.

Tras llevar a cabo este trabajo, los problemas que encontramos son tres, el aburrimiento, la motivación y las relaciones sociales en educación física.

En cuanto al factor aburrimiento, destacamos los resultados del trabajo de Baena-Extremera et al. (2012). En los datos se puede observar este factor diferenciado por sexo, siendo las chicas las que presentan un mayor grado de aburrimiento con respecto a los chicos, al contrario que Castillo, Balaguer, y Duda (2001). Este factor nos da unos resultados no significativos (Tabla 5) al compararlos por el sexo, lo cual es buen resultado ya que el factor aburrimiento no se decanta por ningún grupo en concreto. Sin embargo, como se puede observar en la tabla 4 disminuye el aburrimiento una vez realizadas las sesiones.

En cuanto al problema de la motivación, los datos han presentado un cambio importante significativo con respecto al pre. Esto refleja que se ha producido un cambio mejorando la motivación intrínseca (Tabla 4), y a su vez creando durante las sesiones un deseable clima de tarea, lo que propicia un buen clima motivacional para los alumnos de acuerdo con Moreno-Murcia y Llamas (2007).

Esto ha derivado en actividades orientadas a la tarea, dando como resultado una mejora intrínseca de los alumnos, siendo confirmado por autores tales como Cervelló y Santos-Rosa (2007) y Cury, Biddle, Famose, Sarrazin, Durand, y Goudas (1996). Este cambio motivacional con respecto a 
las sesiones realizadas tienen un resultado positivo, puesto que las actividades propuestas han despertado en los alumnos cierta curiosidad, y a su vez una mejora de la motivación intrínseca.

Por otro lado en la motivación extrínseca y amotivación, se ha producido un cambio significativo reduciéndose ligeramente una vez realizadas las sesiones, como podemos observar (Tabla 4), dando como resultado una posible disminución del ego, puesto que en ocasiones puede estar relacionado. Además puede observarse un cambio significativo por sexo de la motivación extrínseca de un .030 (Tabla 5).

Por tanto, estos datos son de gran interés, ya que la motivación extrínseca está más relacionada con el ego, de acuerdo con autores como Vlachopoulos y Biddle (1997) y Dorobantu y Biddle (1997). Reforzando así nuestra conclusión a cerca de la motivación extrínseca, además afecta de forma negativa puesto que puede derivar en problemas tales como la ansiedad según Ommundsen (2001).

Por último, pero no menos importante, los factores relacionados con las metas sociales han dado unos resultados también significativos. Se puede observar como el factor de relación entre compañeros ha mejorado en el post (Tabla 4), al tratarse de actividades no competitivas y en cierto modo cooperativas, esto ha provocado que los alumnos dejen de lado sus diferencias y se dedicaran de forma conjunta a realizar las actividades propuestas.

Esto también influye en una mejora motivacional como se ha comentado anteriormente y de acuerdo con Guan, Xiang, McBride, y Keating (2013). Por otro lado nuestros resultados en relación al factor de Responsabilidad no han sido significativos. Otros autores como Moreno-Murcia, Coll y Camacho (2007), han realizado investigaciones donde se destaca unos altos resultados de responsabilidad positivos y se sugiere que los docentes desarrollen más metas de responsabilidad en las clases de educación física para conseguir mayores efectos positivos tanto en el aprendizaje como en la participación de los alumnos.

\section{Conclusión}

Es necesario destacar que en este estudio se ha utilizado una muestra poco numerosa, por ello, debemos clasificar estos resultados como preliminares. Para añadir un apoyo a los resultados, las futuras investigaciones deberán utilizar un número mayor de participantes, ya que debemos tener en cuenta que en los estudios cuasi-experimentales existen variables que no pueden controlarse.

A modo de reflexión, cabe destacar el $\alpha$ tan baja que se ha obtenido en el cuestionario Sport Satisfaction Instrument (SSI). En mi opinión, esto puede deberse al contraste de preguntas seguidas, puesto que una trata sobre la diversión y seguidamente sobre el aburrimiento, lo cual puede producir cierta confusión en los alumnos o simplemente que no prestaran la atención suficiente, por ello se ha obtenido una fiabilidad tan baja.

Como conclusión final, destacar que a pesar de las posibles mejoras del trabajo, puesto que se trata de una investigación cuasi-experimental, se han aportado evidencias claras pre post, donde se puede observar que aplicando actividades novedosas e interesantes para los alumnos, puede mejorarse la motivación o reducir el aburrimiento en las aulas.

\section{Referencias}

Anderman, L. H. y Anderman, E. M. (1999). Social predictors of changes in students' achievement goal orientations. Contemporary Educational Psychology, 24(1), 21-37. DOI: 10.1006/ceps.1998.0978

Arias, J. L., Castejón, F. J., y Yuste, J. L. (2013). Propiedades psicométricas de la escala de intencionalidad de ser físicamente activo en Educación Primaria. Revista de Educación.362, 485-505.

Baena-Extremera, A. y Granero-Gallegos, A. (2013) Estudio cuasi-experimental de un programa de supervivencia en el medio natural. Revista Internacional de Medicina y Ciencias de la Actividad Física y el Deporte, 13(51), 551-567.

Baena-Extremera, A., Granero-Gallegos, A. y Ortiz-Camacho, M.M. (2011). Quasi-experimental study of the effect of an Adventure Education programme on classroom satisfaction, physical self-concept and social goals in Physical Education. Psychologica Belgica, 52(4), 386-396. 
Baena-Extremera, A., Granero-Gallegos, A., Bracho-Amador, C., y Pérez-Quero, F. J. (2012). Versión española del Sport Satisfaction Instrument (SSI) adaptado a la educación física. Revista de Psicodidáctica, 17(2), 377-396. DOI: 10.1387/RevPsicodidact.4037

Balaguer, I., Castillo, I., y Duda, J. L. (2007). Propiedades psicométricas de la escala de motivación deportiva en deportistas españoles. Revista Mexicana de Psicología, 24(2), 197-207.

Berrocal, P. F., y Pacheco, N. E. (2005). La Inteligencia Emocional y la educación de las emociones desde el Modelo de Mayer y Salovey. Revista Interuniversitaria de Formación del Profesorado, (54), 63-94.

Brière, N. M., Vallerand, R. J.;Blais, M. R., y Pelletier, L. G. (1995): Développement et validation d'une mesure de motivation intrinsèque, extrinsèque et d'amotivation en contexte sportif: l'Echelle de motivation dans les sports (EMS), International Journal of Sport Psychology, 26, 465-489.

Castillo, I., Balaguer, I., y Duda, J. L. (2001). Perspectivas de meta de los adolescentes en el contexto académico. Psicothema, 13(1), 79-86.

Cervelló, E. M., y Santos-Rosa, F. (2007). Motivación en las clases de Educación Física: un estudio de la perspectiva de las metas de logro en el contexto educativo. Revista de Psicología del Deporte, 9(12), 51-70.

Cury, F., Biddle, S., Famose, J. P., Sarrazin, P., Durand, M., y Goudas, M. (1996). Personal and situational factors influencing intrinsic interest of adolescent girls in school physical education: A structural equation modelling analysis. Educational Psychology, 16(3), 305-315. DOI: 10.1080/0144341960160307

Dorobantu, M., y Biddle, S. (1997). The influence of situational and individual goals on the intrinsic motivation of adolescents towards Physical Education. The European Yearbook of Sport Psychology, 1, 148-165.

Duda, J. L. y Nicholls, J. G. (1989). The task and ego orientation in sport questionnaire: Psychometric properties. Unpublished manuscript.

Duda, J. L. y Nicholls, J. G. (1992). Las dimensiones de la motivación de logro en el trabajo escolar y el deporte. Diario de Psicología de la Educación, 84 (3), 290-299.

Granero-Gallegos, A., Baena-Extremera, A., Gómez-López, M., y Abraldes, J. A. (2013). Análisis preliminar exploratorio del "Sport Motivation Scale (SMS)" adaptado a la Educación Física. Espiral. Cuadernos del Profesorado, 6(12), 3-14. DOI: 10.25115/ecp.v6i12.953

Guan, J., Xiang, P., y McBride, R. (2005). Reliability and Validity Evidence for the Social Goal Scale-Physical Education in High School Settings. Research Quarterly for Exercise and Sport, 76, A51.

Guan, J., Xiang, P., McBride, R., y Keating, X. D. (2013). Achievement goals, social goals, and students' reported persistence and effort in high school athletic settings. Journal of Sport Behavior, 36(2), 149.

Inglés, C. J., Martínez-González, A. E., Torregrosa, M. S., García-Fernández, J. M., y Ruíz-Esteban, C. M. (2011). Conducta prosocial y autoconcepto en una muestra de estudiantes españoles de Educación Secundaria Obligatoria. Revista de Psicodidáctica, 17(1), 136-141.

Ministerio de Educación, Cultura y Deporte (2014). Real Decreto 126/2014, de 28 de Febrero, por el que se establece el currículo básico de la Educación Primaria. BOE 52, de 1 de Marzo de 2014, 44-49.

Moreno-Murcia, J. A., González Cutre-Coll, D., y Sicilia, Á. (2007). Metas sociales en las clases de educación física. Análisis y Modificación de Conducta, 33(149), 351-368.

Moreno-Murcia, J. A., y Llamas, L. S. (2007). Predicción de la importancia concedida a la educación física según el clima motivacional y la motivación autodeterminada en estudiantes adolescentes. Enseñanza, $25,11,138-155$.

Nicholls, J. G., Cheung, P. C., Lauer, J., y Patashnick, M. (1989). Individual differences in academic motivation: Perceived ability, goals, beliefs, and values. Learning and Individual Differences, 1(1), 63-84. DOI: 10.1016/1041-6080(89)90010-1

Ommundsen, Y. (2001). Pupils Affective Responses in Physical Education Classes: the Association of Implicit Theories of the Nature of Ability and Achievement Goals. European Physical Education Review, 7(3), 219-242. DOI: $10.1177 / 1356336 \mathrm{X} 010073001$

Ryan, R. y Deci, E. L. (2000). La Teoría de la Autodeterminación y la Facilitación de la Motivación Intrínseca, el Desarrollo Social, y el Bienestar. American Psychologist, 55(1), 68-78. DOI: 10.1037/0003066X.55.1.68 
Vlachopoulos, S. y Biddle, S. J. (1997). Modeling the relation of goal orientations to achievement-related affect in physical education: Does perceived ability matter? Journal of Sport y Exercise Psychology 19,169187. DOI: $10.1123 /$ jsep.19.2.169

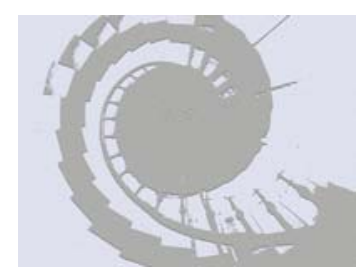

